\title{
Comparación del tratamiento médico y quirúrgico en pacientes con obesidad grado III (obesidad mórbida)
}

\author{
Attila Csendes J, Patricio Burdiles P, Karin Papapietro V, \\ Ana María Burgos L. \\ Review of the results of medical and \\ surgical treatment of morbid obesity
}

This is a review of publications comparing the results of medical and surgical treatment of morbid obesity. An overall conclusions is that the frequency of cardiovascular complications or cancer is higher among patients receiving medical treatment. Surgical treatment is associated with a better weight loss, reduction in complications and quality of life. Mortality risk decreases significantly after surgical treatment, when compared with patients receiving medical therapy. Therefore, management of morbid obesity should be carried out by multidisciplinary teams with experience on gastrointestinal surgery. In this way the complications and mortality of bariatric surgery would be minimized (Rev Méd Chile 2009; 137: 559-66).

(Key w ords: Bariatric surgery; Obesity, morbid; Survival rate)

Recibido el 28 de marzo, 2008. Aceptado el 28 de julio, 2008.

Departamento de Cirugía, Hospital Clínico de la Universidad de Chile. Santiago, Chile.

L a obesidad mórbida se ha convertido en una —verdadera epidemia del siglo XXI. Estadísticas recientes del Ministerio de Salud indican que cerca de 1,3\% de la población chilena presenta esta enfermedad, lo que equivale que cerca de 120.000 a 150.000 chilenos lo presentan en la actualidad $^{1}$. Hay consenso en todos los centros médicos dedicados a este tema que la cirugía es el tratamiento de elección en estos pacientes, ya que el tratamiento médico es ineficaz a este nivel de obesidad. ¿En qué se basa esta presunción?, ¿qué estudios demuestran estas diferencias? En el pre-

Correspondencia a: Dr. Attila Csendes, FACS (Hon). Departamento de Cirugía, Hospital Clínico J.J. Aguirre. Santos Dumont 999, Santiago Chile. Fono: 56-2-7774387. Fax: 56-2-7775043. E mail: acsendes@redclinicauchile.cl sente artículo hemos revisado 27 publicaciones, desde 1980 hasta la fecha (agosto 2007), que se refieren a diferentes aspectos del tratamiento médico y quirúrgico en pacientes con obesidad mórbida. Esta búsqueda no es un metaanálisis, sino una revisión de toda la literatura anglosajona que se refiere a tratamiento médico o quirúrgico de la obesidad mórbida.

I. Estudios prospectivos y randomizados comparando tratamiento médico o quirúrgico en pacientes con obesidad mórbida

Hay solo una publicación hasta ahora realizada de manera prospectiva y aleatoria ${ }^{2}$.

En este estudio danés se compararon 30 obesos mórbidos sometidos a tratamiento médico por 2 años con 27 pacientes sometidos a dieta y gastroplastía horizontal, evaluados por 2 años. La

A RTÍCULO

DE REVISIÓN 
cirugía consistió en la confección de una bolsa gástrica de $50 \mathrm{ml}$ con una boca de $11 \mathrm{~mm}$, comunicado hacia el estómago distal (esta operación no se practica actualmente). La dieta contenía 900 calorías. A los 2 años, la pérdida de peso con tratamiento médico al inicio fue de $10 \%$, recuperándose el peso a los 2 años. Con la cirugía, se obtuvo una baja de peso de $30 \%$, lo que fue estadísticamente muy significativo $(p<0,05)$.

Otra publicación reciente de tipo teórico, que empleó un modelo de análisis de decisión, que es una herramienta útil para determinar la estrategia óptima de tratamiento ${ }^{3}$. Mediante este análisis de decisión, se simuló un estudio comparando los resultados de cirugía versus tratamiento médico consistente en dieta y ejercicios, con el objeto de determinar cuál estrategia resulta en una mayor expectativa de vida. Con tratamiento médico a 2 años se obtuvo una reducción de peso en $20 \%$ de los casos, recuperándose el peso pretratamiento en $95 \%$ de ellos. Con la cirugía $80 \%$ de los casos perdió peso, recuperando el peso preoperatorio en solo $5 \%$ ( $p<0,001)$. Se obtuvo una ganancia de vida de 4,1 años, lo que equivale a $11 \%$ de aumento de sobrevida con la cirugía.

II. Resultados del tratamiento médico exclusivo en pacientes con obesidad mórbida (estudios no aleatorios)

El primer estudio se publicó en $1980^{4}$ en que se siguió a 200 hombres con obesidad mórbida, seguimiento promedio de 7,6 años. El peso promedio fue de $143 \mathrm{~kg}$. Al principio hubo una discreta baja de peso con la dieta, pero todos recuperaron peso antes de los 2 años. Hubo 50 fallecidos durante el seguimiento, básicamente por enfermedades cardiovasculares (54\%) y cáncer (8\%). La probabilidad porcentual de exceso de fallecimiento calculado por décadas de edad, comparado con la mortalidad de los hombres norteamericanos en total, fue de $1.100 \%$ (11 veces mayor) entre los 25 y 34 años y de $500 \%$ (5 veces mayor) entre los 35 y 44 años.

El segundo estudio, en 1990, evaluó el riesgo de enfermedad coronaria en mujeres obesas ${ }^{5}$. El seguimiento de 115.886 obesas hasta 8 años demostró 605 eventos coronarios, 306 infartos, 216 anginas y 83 muertes, demostrando que con el aumento de peso el riesgo de infarto se incrementó 1 a 3,3 veces.
Posteriormente en 2 estudios del grupo sueco de estudio de obesidad 6,7 , se demostró que la morbilidad subió dramáticamente con la obesidad, en especial con el aumento de la grasa visceral ${ }^{6}$. El incremento de la incidencia de cáncer en hombres se observó en colon y próstata y en mujeres en vesícula, mama y útero ${ }^{7}$. También se observó que $90 \%$ de los pacientes sometidos a cualquier tipo de tratamiento médico recuperaron el peso inicial al año de observación. Bonelli y cols $^{8}$ evaluaron 264 obesos mórbidos tratados médicamente durante 7 años. La mortalidad fue 6,9 veces mayor en mujeres y 4,3 veces mayor en hombres entre 25 y 54 años comparados con la población general.

Posteriormente, en $1993^{9}$, se analizó la relación entre la pérdida de peso en obesos y una mayor longevidad. Este artículo resumió las observaciones de 6 publicaciones entre 1951 y 1990, demostrando en 5 de ellos una reducción de la mortalidad entre $6 \%$ y $50 \%$ con un promedio de $30 \%$, por enfermedades cardiovasculares en especial el infarto del miocardio al bajar de peso.

III. Comparación del tratamiento médico y quirúrgico respecto a baja de peso y mejoría de las comorbilidades a largo plazo (estudios no aleatorios). Hay 11 artículos que se refieren específicamente a este aspecto (Tabla 1). Foley en $1992^{10}$ evaluó a 249 pacientes obesos mórbidos hipertensos sometidos a bypass gástrico con Y-de-Roux y a gastroplastia vertical. La hipertensión arterial se resolvió en $66 \%$, que se relacionó con la pérdida de peso, ya que si esta pérdida era mayor, la reducción de la hipertensión fue de $70 \%$. Pories y cols $^{11}$ en 1995 publicaron un artículo trascendental en cirugía bariátrica. Estos autores siguieron a 608 operados sometidos a bypass gástrico con Yde-Roux hasta 14 años de la operación. Demostraron por primera vez que la baja de peso se mantiene a varios años de la operación, y lo que fue más trascendente, que la diabetes se resolvió en $83 \%$ y la resistencia a la insulina en $99 \%$. MacDonald y cols ${ }^{12}$ reportaron a 78 pacientes no operados seguidos por 6 años y 154 pacientes sometidos a bypass gástrico con Y-de-Roux. El porcentaje de diabéticos en el grupo médico subió de $56 \%$ a $87 \%$, mientras que en el grupo quirúrgico se resolvió en 90\%. Karlsson y cols evaluaron a 487 pacientes no operados compara- 
Tabla 1. Cambios en el peso y la comorbilidad en pacientes con obesidad mórbida, comparando tratamiento médico o quirúrgico

\begin{tabular}{|c|c|c|c|}
\hline Autor & Tratamiento médico & Tratamiento quirúrgico & $\begin{array}{l}\text { Comentarios de } \\
\text { resultados quirúrgicos }\end{array}$ \\
\hline $\begin{array}{l}\text { Estudios randomizados } \\
\text { Anderssen (1984) }\end{array}$ & $\begin{array}{l}\text { Baja de peso de } 10 \% \text { en } \\
30 \text { pacientes }\end{array}$ & $\begin{array}{l}\text { A } 2 \text { años baja de peso de } 30 \% \\
\text { en } 30 \text { operados }(p<0,05)\end{array}$ & $\begin{array}{l}\text { Único estudio prospectivo } \\
\text { y randomizado }\end{array}$ \\
\hline $\begin{array}{l}\text { Estudios no } \\
\text { randomizados } \\
\text { Foley (1992) }\end{array}$ & & 249 obesos mórbidos, hipertensos & $\begin{array}{l}\text { Baja peso resolvió } \\
\text { hipertensión en } 66 \%\end{array}$ \\
\hline Pories (1995) & & $\begin{array}{l}608 \text { operados seguidos a } 14 \text { años. } \\
146 \text { diabéticos y } 152 \text { con } \\
\text { insulino-resistencia }\end{array}$ & 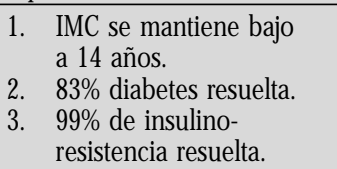 \\
\hline Mac Donald (1997) & $\begin{array}{l}78 \text { seguidos } 6,2 \text { años. } \\
\text { Diabetes aumento de } \\
56 \% \text { a } 87 \%\end{array}$ & $\begin{array}{l}154 \text { diabéticos operados } \\
\text { seguidos } 9 \text { años }\end{array}$ & $\begin{array}{ll}\text { 1. } & \text { Pérdida exceso peso } \\
60 \% \text { a } 14 \text { años. } \\
\text { 2. } \\
\text { Diabetes controlada en } \\
90 \% .\end{array}$ \\
\hline Karlsson (1988) & $\begin{array}{l}487 \text { seguidos } 2 \text { años = IMC } \\
\text { se mantuvo igual que } \\
\text { preop }(40 \text { a 40) }\end{array}$ & $\begin{array}{l}487 \text { operados seguidos } 2 \text { años. } \\
\text { IMC bajó de } 42 \text { a } 31\end{array}$ & $\begin{array}{l}\text { Calidad vida mejoró } \\
\text { sustancialmente con cirugía. }\end{array}$ \\
\hline Torgerson (2001) & $\begin{array}{l}\text { Baja de peso a } 2 \text { años en } \\
\text { no operados de } 0,5 \mathrm{~kg}\end{array}$ & $\begin{array}{l}\text { Baja de peso a } 2 \text { años } \\
\text { en operados de } 28 \mathrm{~kg}\end{array}$ & $\begin{array}{ll}\text { 1. } & \text { Diabetes se redujo } 32 \\
\text { veces comparado con no } \\
\text { operados. } \\
\text { 2. Hipertensión se redujo } \\
\text { 2,6 veces. } \\
\text { 3. Número de días perdidos } \\
\text { por licencias es doble } \\
\text { en tratamiento médico. }\end{array}$ \\
\hline Agren (2002) & $\begin{array}{l}962 \text { no operados seguidos } \\
6 \text { años peso aumenta en } \\
0,9 \% \text {. Estadía hospitalaria } \\
\text { total } 6,9 \text { días }\end{array}$ & $\begin{array}{l}962 \text { operados seguidos } 6 \text { años. } \\
\text { Baja de peso de }-17 \% \text {. Estada } \\
\text { hospitalaria total de } 23,4 \text { días }\end{array}$ & $\begin{array}{l}\text { 1. Costo promedio mayor } \\
\text { después tratamiento } \\
\text { quirúrgico. } \\
\text { 2. Aumento costo es } \\
\text { moderado considerando } \\
\text { alto número de } \\
\text { beneficios operación. }\end{array}$ \\
\hline Sjöström (2004) & $\begin{array}{l}627 \text { no operados seguidos } \\
10 \text { años. Peso aumentó } 1,6 \%\end{array}$ & $\begin{array}{l}641 \text { operados seguidos } 10 \text { años. } \\
\text { Baja de peso de } 16 \%\end{array}$ & $\begin{array}{l}\text { Cirugía, la baja de peso } \\
\text { mantenida mejora calidad } \\
\text { de vida y comorbilidades } \\
\text { (diabetes, hiper-trigliceri- } \\
\text { demia) }\end{array}$ \\
\hline Christou (2004) & $\begin{array}{l}5.746 \text { no operados y } \\
\text { seguidos } 5 \text { años. Costo total } \\
\text { tratamiento médico } \\
11.854 \text { dólares. } \\
\text { Hospitalizaciones } \\
35.411 \text { veces. } \\
\end{array}$ & $\begin{array}{l}1.035 \text { operados seguidos } 5 \text { años. } \\
\text { Costo total tratamiento quirúrgico } \\
8.813 \text { dólares. Hospitalizaciones } \\
2,840 \text { veces }\end{array}$ & $\begin{array}{l}\text { 1. Baja de peso mantenida de } \\
67 \% \text {. } \\
\text { 2. Comorbilidades que } \\
\text { bajan cardiovasculares, } \\
\text { diabetes, respiratorias, } \\
\text { infecciones. }\end{array}$ \\
\hline Buchwald (2004) & & $\begin{array}{l}22.094 \text { pacientes operados seguidos } \\
2 \text { años. Perdida de exceso de peso } \\
\text { de } 61 \%\end{array}$ & $\begin{array}{l}\text { Diabetes resuelta en } 75 \% \\
\text { Hipertensión resuelta en } 68 \% \\
\text { Apnea sueño resuelta en } 86 \% \\
\end{array}$ \\
\hline Ryden (2006) & $\begin{array}{l}2.037 \text { no operados seguidos } \\
\text { a } 10 \text { años. Aumento peso } 1,6 \%\end{array}$ & $\begin{array}{l}2.010 \text { operados seguidos a } 10 \text { años. } \\
\text { Disminución peso de } 38 \% \text {. }\end{array}$ & \\
\hline Sjöström (2007) & $\begin{array}{l}2.037 \text { no operados seguidos } \\
11 \text { años. Aumento } 2 \% \text { peso. }\end{array}$ & $\begin{array}{l}2.010 \text { operados seguidos a } 15 \text { años. } \\
\text { Disminución peso de } 25 \% \\
\text { con bypass. }\end{array}$ & $\begin{array}{l}\text { Mejoría de diabetes, enferme- } \\
\text { dades cardiovasculares, } \\
\text { apnea del sueño, } \\
\text { osteoarticulares. }\end{array}$ \\
\hline
\end{tabular}

A RTÍCULO 
dos con 487 operados $^{13}$, seguidos a 2 años plazo. El IMC promedio de los no operados se mantuvo exactamente igual después de 2 años (IMC de 40), mientras que el grupo quirúrgico disminuyó el IMC de 42 a 31, acompañado de una mejoría sustancial de la calidad de vida. Torgerson y cols ${ }^{14}$ señalaron que la baja de peso en obesos mórbidos no operados a 2 años fue sólo de $0,5 \mathrm{~kg}$, mientras que en los operados fue de $28 \mathrm{~kg}$. La reducción de la diabetes y la hipertensión arterial fue muy importante y demostraron que el número de días perdidos por licencias médicas fue el doble en obesos tratados médicamente, mientras que la pensión de invalidez fue 2 veces mayor entre 2 y 4 años después de la operación.

Agren y cols ${ }^{15}$ compararon 962 no operados con 962 operados y seguidos por 6 años. En los controles hubo un aumento de peso de 0,9\%, mientras que en los operados hubo una disminución de $17 \%$ del peso preoperatorio ( $p<0,0001$ ). Este estudio demostró que los días totales de hospitalización fue de 23,4 días en los operados y de 6,9 días con tratamiento médico $(\mathrm{p}<0,0001)$ comparando paciente con paciente. También el costo promedio fue mayor entre los operados comparados con los no operados. Sin embargo, los autores enfatizaron que el aumento de costo debe considerarse en el contexto de los beneficios del tratamiento quirúrgico; baja de peso mantenida, disminución de los efectos adversos cardiovasculares y mejoría de la calidad de vida. Sjöström y cols $^{16}$ analizaron 627 pacientes no operados con 641 operados seguidos 2 y 10 años. Este estudio merece ser destacado por su metodología: análisis prospectivo no randomizado, pero pareado en 18 variables como edad, género, peso, circunferencia cintura, etc. El seguimiento fue el más largo reportado hasta esa fecha (10 años). El grupo no operado mostró un aumento de peso de 1,6\% mientras que el grupo quirúrgico a 10 años mantuvo una disminución de peso de $16 \%$ ( $p$ $<0,01$ ). Este artículo incluyó 3 técnicas quirúrgicas, observando los mejores resultados después de bypass gástrico con Y-de-Roux, y los peores resultados con el banding gástrico (baja de peso a 10 años de $25 \%$ y $13 \%$, respectivamente). El grupo quirúrgico mejoró notablemente en cuanto a diabetes, hipertrigliceridemia e hiperuricemia y no hubo diferencias en los niveles de colesterol y de la hipertensión arterial. Christou y cols ${ }^{17}$ compara- ron 5.746 no operados con 1.035 obesos mórbidos operados y seguidos a 5 años. No se informa sobre el peso en los no operados, pero los costos totales (en dólares canadienses) fueron mayores y el número de hospitalizaciones fue mayor entre los no operados comparados con el tratamiento quirúrgico. El grupo operado presentó una disminución del exceso de peso de $25 \%$ a 2 años y mantuvo una reducción de las complicaciones cardiovasculares, endocrinológicas, diabetes, respiratorias e infecciosas. Buchwald y cols ${ }^{18}$ analizaron los resultados de 22.094 operados seguidos por 2 años, comparando 4 operaciones. La pérdida de exceso de peso fue de $61 \%$, siendo mayor para la derivación biliopancreática y la menor para la banda gástrica. La diabetes se resolvió en $75 \%$, la hipertensión en $61 \%$ y la apnea de sueño en $86 \%$. Ryden y cols ${ }^{19}$ evaluaron a 2.037 pacientes no operados con 2.010 obesos mórbidos operados y seguidos a 4 años. El grupo control se mantuvo en el peso e incluso subió 1,6\% comparado con el peso inicial. El grupo sometido a bypass gástrico demostró una reducción de peso de $38 \%$, con mejoría cardiovascular evidente.

En un estudio reciente del grupo sueco de obesidad (SOS), Sjöström y cols ${ }^{20}$ publicaron un estudio de seguimiento postoperatorio hasta 15 años, comparando 2.037 obesos no operados con 2.050 operados. En este estudio prospectivo y controlado, el grupo control mostró una variación de peso menor a $2 \%$ mientras que el grupo quirúrgico mostró una reducción de peso de $25 \%$ a 15 años con el bypass gástrico, comparado con una baja de peso de $14 \%$ con la banda gástrica. Hubo una mejoría de las comorbilidades como diabetes, apnea del sueño, problemas osteoarticulares y cardiovasculares.

Hay además 3 artículos no comparativos que señalaron resultados similares. Benotti y Forse ${ }^{21}$ en 1995 comentaron que la baja de peso con tratamiento médico intensivo fue de $10 \mathrm{~kg}$ al año en 3 reportes, mientras que 2 estudios citaron una baja de peso a 10 años de 9,5 $\mathrm{kg}$ en mujeres con tratamiento médico continuo. Sin embargo, después de tratamiento quirúrgico la calidad de vida y las comorbilidades mejoraron en forma muy significativa. Martin y cols ${ }^{22}$ en una revisión concluyeron que ningún tratamiento médico de la obesidad mórbida ha mostrado una pérdida de peso estable por 5 años, ni siquiera en el $10 \%$ de los tratados. 
Buchwald ${ }^{23}$ en un completo análisis publicado en 2005 concluyó los siguientes puntos más importantes: a) la cirugía baríatrica es más efectiva que el tratamiento médico de la obesidad, b) que es aconsejable que todos los candidatos a cirugía hayan tenido un tratamiento médico previo, c) deben tener una evaluación completa, d) cirugía de adolescentes solo en centros con experiencia y e) la cirugía es costo efectiva a los 4 años plazo.

IV. Comparación de la mortalidad a largo plazo del tratamiento médico y quirúrgico (estudios no randomizados). Un punto muy importante es determinar si el tratamiento quirúrgico, además de obtener una reducción de peso, una mejoría de la calidad de vida y una mejoría notable de las comorbilidades, es capaz de lograr un cambio o no en la mortalidad alejada y por lo tanto en la sobrevida de los pacientes. En la Tabla 2 señalan los autores que han evaluado este aspecto, MacDonald y cols ${ }^{12}$ demostraron que la mortalidad durante el periodo de seguimiento de los pacientes con obesidad mórbida fue de $28 \%$ en los no operados y de $9 \%$ en los operados, es decir, se obtuvo una reducción de $67 \%$ de la mortalidad, en especial de origen cardiovascular, después de la

Tabla 2. Comparación de la mortalidad a largo plazo del tratamiento médico y quirúrgico en pacientes con obesidad mórbida (estudios no randomizados)

\begin{tabular}{|c|c|c|c|}
\hline Autor & Tratamiento médico & Tratamiento quirúrgico & $\begin{array}{l}\text { Comentario de los } \\
\text { resultados quirúrgicos }\end{array}$ \\
\hline Mac Donald (1997) & 78 no operados seguidos 6,2 años & $\begin{array}{l}154 \text { operados seguidos } \\
9 \text { años }\end{array}$ & $\begin{array}{l}\text { Mortalidad } \\
28 \% \text { no operados } \\
9 \% \text { operados }\end{array}$ \\
\hline Christou (2004) & 5.746 no operados seguidos 5 años & $\begin{array}{l}1.035 \text { operados seguidos } \\
5 \text { años }\end{array}$ & $\begin{array}{l}\text { Mortalidad } \\
6,17 \% \text { no operados } \\
0,68 \% \text { operados }\end{array}$ \\
\hline Flum (2004) & Sobrevida a 10 años $80 \%$ & $\begin{array}{l}\text { Sobrevida } 10 \text { años } 91,2 \% \\
(0,004)\end{array}$ & $\begin{array}{l}\text { Mortalidad } \\
\text { A } 15 \text { años de seguimiento: } \\
\text { 16,3\% no operados } \\
11,5 \% \text { operados }\end{array}$ \\
\hline Sowemino (2007) & 112 no operados & $\begin{array}{l}908 \text { operados. Hay una } \\
\text { disminuciónde } 82 \% \\
\text { de la mortalidad. }\end{array}$ & $\begin{array}{l}\text { Mortalidad } \\
14.3 \% \text { no operados } \\
2,9 \% \text { operados } \\
(\mathrm{p}<0,001) \\
\end{array}$ \\
\hline Dixon (2006) 4 estudios & $\begin{array}{l}\text { 1. Suecia } 2.000 \text { no operados. } \\
\text { 2. Australia } 2.000 \text { no operados } \\
\text { 3. UTAH } 817 \text { no operados } \\
\text { 4. Padova } 821 \text { no operados }\end{array}$ & $\begin{array}{l}2.000 \text { operados } \\
1.468 \text { operados } \\
817 \text { operados } \\
821 \text { operados }\end{array}$ & $\begin{array}{l}\text { Mortalidad } \\
\text { Reducción de } 31.6 \% \text { con } \\
\text { cirugía (p }<0,01 \text { ) } \\
73 \% \text { reducción } \\
\text { mortalidad con operación } \\
40 \% \text { reducción de } \\
\text { mortalidad con operación } \\
62 \% \text { reducción de } \\
\text { mortalidad con operación }\end{array}$ \\
\hline Adams (2007) & 7.925 no operados & 7.925 bypass seguidos 7 años & $\begin{array}{l}\text { Reducción de mortalidad } \\
\text { de } 40 \% \text { en el grupo } \\
\text { quirúrgico }\end{array}$ \\
\hline Sjöström (2007) & 2.037 no operado, seguidos 11 años. & $\begin{array}{l}2.010 \text { operados seguidos } \\
11 \text { años. }\end{array}$ & $\begin{array}{l}\text { Mortalidad } 6,3 \% \text { grupo } \\
\text { médico. } 5,0 \% \text { grupo } \\
\text { quirúrgico }(p<0,04)\end{array}$ \\
\hline
\end{tabular}

A RTÍCULO 
cirugía. Christou y cols ${ }^{17}$ en un seguimiento de 5 años de grupo médico y quirúrgico observaron una mortalidad de 6,17\% en el grupo médico y de $0,68 \%$ en el grupo quirúrgico, con una reducción de $89 \%$ de la mortalidad en los operados. Flum y cols $^{24}$ comunicaron una mortalidad, a 15 años de seguimiento, de $16,3 \%$ en el grupo médico y de $11,5 \%$ en el grupo quirúrgico. La probabilidad de fallecimiento fue $33 \%$ menor para los operados comparado con los pacientes no operados. Sowemino y cols ${ }^{25}$ compararon 112 no operados y 908 sometidos a cirugía bariátrica. La mortalidad de los no operados fue de $14,3 \%$ y de los operados de $2,9 \%(\mathrm{p}<0,001)$, con una reducción de la mortalidad de $82 \%$ entre los operados. Finalmente Dixon $^{26}$ resumió las presentaciones de 4 grupos durante el Congreso de Obesidad en 2006:

a) El grupo sueco analizando 2.000 no operados, con 2.000 pacientes operados, observó una reducción de 31,6\% ( $\mathrm{p}<0,01)$ de la mortalidad por patología cardiovascular y cáncer entre los no operados.

b) El grupo de Australia evaluó 2.000 obesos mórbidos no operados con 1.468 sometidos a banda gástrica. Hubo 5 fallecidos entre los operados y 225 fallecidos entre los no operados, con una reducción de $73 \%$ de la mortalidad.

c) El grupo de Utah comparó 812 no operados con 812 sometidos a bypass gástrico, con controles hasta 18 años, demostrando una reducción de la mortalidad de $40 \%$ por enfermedades cardiovasculares, diabetes y cáncer.

d) El grupo de Padova reportó 821 no operados y 821 pacientes sometidos a banda gástrica, observando una reducción de $62 \%$ de la mortalidad entre los operados.

En un estudio reciente de agosto de 2007, se comparó la mortalidad a largo plazo (7 años) de 7.925 obesos mórbidos no operados con 7.925 sometidos a bypass gástrico ${ }^{27}$. Se apreció una disminución de $40 \%$ de la mortalidad en el grupo quirúrgico comparado con el control, es especial para enfermedad coronaria (56\% reducción), la diabetes (92\% reducción) y cáncer (60\% reducción de mortalidad). Otras causas de mortalidad como accidentes y suicidios aumentaron en $58 \%$ en el grupo quirúrgico comparado con el control. El estudio sueco sobre obesidad ${ }^{20}$ mostró los resulta- dos hasta 15 años de un grupo control no operado de 2.037 pacientes comparando con 2.010 operados. Se apreció una mortalidad de $6,3 \%$ en el grupo médico y de $5 \%$ en el grupo quirúrgico, en especial por enfermedades cardiovasculares y cáncer $(\mathrm{p}<0,04)$.

\section{CONCLUSIONES}

1. Hay solo un estudio prospectivo y randomizado publicado en 1984 comparando dieta y ejercicios con dieta + cirugía en pacientes con obesidad mórbida, observando a 2 años un resultado significativamente mejor con la cirugía comparada con tratamiento médico. La técnica quirúrgica utilizada ha sido abandonada desde hace muchos años, por lo que su validez es relativa. Este estudio sugiere que la cirugía gástrica resulta en una muy buena baja de peso en pacientes muy obesos y en los cuales todas las otras medidas terapéuticas han fallado ${ }^{28}$.

2. Hay 11 estudios que han evaluado el riesgo de complicaciones y la sobrevida a largo plazo de obesos mórbidos tratados médicamente. En todos se demostró que la mortalidad aumenta en forma importante ya sea por eventos cardiovasculares, diabetes o cáncer. La mayor probabilidad de fallecer ocurrió en los jóvenes entre 25 y 34 años, comparados con la población general.

También se destacó el hecho que al bajar de peso con tratamiento médico, se apreció una reducción de la mortalidad.

3. Hay 11 estudios que analizaron la evolución del peso y de las comorbilidades, ya sea con tratamiento médico o quirúrgico en pacientes con obesidad mórbida. Los resultados demostraron lo siguiente:

a. Con tratamiento médico prácticamente no hubo variación del peso con un seguimiento entre 2 y 15 años, incluso apreciando una leve alza del peso inicial.

b. En todos los estudios el tratamiento quirúrgico logró una importante baja de peso, que incluso se mantiene hasta 14 años después de la cirugía.

c. El resultado más espectacular fue la curación o resolución de la diabetes entre $80 \%$ y 
90\% y de la resistencia a la insulina en 95\%, que nunca se logró con tratamiento médico. d. La calidad de vida mejoró notablemente con el tratamiento quirúrgico en todos los trabajos analizados.

4. El riesgo de mortalidad ya sea por tratamiento médico o quirúrgico ha sido evaluado por 10 diferentes grupos, concluyendo todos sin distinción, que después de la cirugía por obesidad mórbida se observó una significativa disminución de la mortalidad comparado con tratamiento médico que va entre $30 \%$ y $80 \%$ : a) la mayor reducción de la mortalidad se apreció después del bypass gástrico comparado con otras técnicas como la banda gástrica o la gastrectomía vertical. b) la reducción de la mortalidad ocumió principalmente por una disminución de la patología cardiovascular, diabetes y cáncer.

Es muy difícil en la actualidad plantear con todos estos datos, un estudio prospectivo y randomizado comparando tratamiento médico versus cualquier técnica quirúrgica actual laparoscópica. Éticamente nosotros no lo podemos plantear, ya que las evidencias existentes son tan contundentes a favor de la cirugía en pacientes con obesidad mórbida, que no justifica tener un grupo médico o control para obtener los mismos resultados que ya se han reportado.

\section{REFERENCIAS}

1. PizarRo T. Estadísticas sobre sobrepeso y obesidad en la población chilena. Ministerio de Salud 2002.

2. Andersen T, Backer OG, Stokholm KH, QuaAde F. Randomized trial of diet and gastroplasty compared with diet alone in morbid obesity. $\mathrm{N}$ Engl J Med 1984; 310: 352-6.

3. Patterson EJ, Lubach OR, Swanstiön L A companison of diet and exercise therapy versus laparoscopic Roux-enY gastric bypass surgery for morbid obesity. A decision analysis model. J Am Coll Surg 2003; 196: 379-84.

4. Drenick EJ, Gurunanjappa S, Seltzer F, Johnson DG. Excessive mortality and causes of death in morbidly obese men. JAMA 1980; 243: 443-5.

5. Manson JE, Colditz GA, Stampfer MJ, Wimett WC, Rosner B, MONSON RR ET AL. A prospective study of obesity and risk of coronoary heart disease in women. N Engl J Med 1990; 322: 882-9.

6. SјöSTRÖM LV. Morbidity of severity obese subjects. Am J Clin Nut 1992; 55: 508S-515S.
5. En las guías clínicas recientes del NIH se reconoce y se recomienda el tratamiento quirúrgico de pacientes con obesidad severa ${ }^{29}$. La misma conclusión es la señalada por la revisión Cochrane ${ }^{28}$.

En suma, todas las evidencias científicas aunque limitadas, sugieren que el tratamiento quirúrgico es muy superior al tratamiento médico en pacientes con obesidad mórbida. La baja de peso después de cirugía es mayor comparada con tratamiento convencional, y los resultados se mantienen hasta por lo menos 15 años. Además, la reducción de peso se asocia a reducciones de la comorbilidad como diabetes e hipertensión. Sin embargo, la cirugía se asocia a eventuales efectos adversos y a la posibilidad de mortalidad postoperatoria, que debe ser evaluada en su contexto. Los datos presentados en esta revisión entrega elementos de juicio para médicos, autoridades de salud e Isapres en el proceso de toma de decisiones en esta patología $20,26,28,29$.

Los pacientes deberán ser siempre analizados por un equipo multidisciplinario, incluyendo nutriólogos, nutricionistas, psiquiatras, psicólogos y cirujanos para decidir en conjunto el tratamiento quirúrgico. De esta manera se asegura al paciente un control ético y científico de su tratamiento.

7. SjöSTRÖm LV. Mortality of severely obese subjects. Am J Clin Nut 1992; 55: 516S-523S.

8. Bonem R, Isernia C, Dibiase G, Contaldo F. Mortality rate, causes and predictive factors in death in severely obese patients. Int J Vit Nutr Res 1998; 58: 343-50.

9. WiLAMASON DF, PAMUK ER. The association between weight loss and increased longevity. Ann Intern Med 1993; 119: 731-6.

10. Foley EF, Benotti PN, Borlase BC, Blackburn G. Impact of gastric restrictive surgery on hypertension in the morbidly obese. Am J Surg 1992; 163: 294-7.

11. Pories WJ, Swanson MS, MacDonald KG, Long SB, MorRIS PG, BRown BM ET AL. Who would have thought it? An operation proven to be the most effective therapy for adult-onset diabetes mellitus. Ann Surg 1995; 222: 339-52.

12. Mac Donald KG, Long SD, Swanson MS, Brown BM, Morris P, Dohm GL, Pories WJ. The gastric bypass operation reduce the progression and mortality of non-insulin-depended diabetes mellitus. J Gastrointest Surg 1997; 1: 213-20.

A Rtíc U LO 
13. KarLSSON J, SjöSTRÖM L, SuLuVAN M. Swedish obese subjects (SOS). An intervention study of obesity. Two-year follow up of health-related quality of life (HRQL) and eating behaviour after gastric surgery for severe obesity. Int J Obes 1988; 22: 113-26.

14. TORGERSON JS, Sjöström L The Swedish obese subjects (SOS) study: rationale and results. Inter J Obesity 2001; 25: 52-4.

15. Agren G, Narbro K, Jonsson E, Naslund I, Sjöström L, Peltonen M. Cost of in-patient case over 7 years among surgically and conventionally treated obese patients. Obes Res 2002;10:1276-1283.

16. Sjöström L, Luidroos AK, Peltonen M, Torgenson J, Bouchard C, Carlsson B et al. Lifestyle, diabetes and cardiovascular risk factor 10 years after bariatric surgery. N Engl J Med 2004; 351: 2683-93.

17. Christou NV, Sampalis JS, Liberman M, Cook D, Auger S, Mclean APH, MacLEan LD. Surgery decreases longterm mortality, morbidity and Health care use in morbidly obese patients. Ann Surg 2004; 240: 41624.

18. Buchwald H, Avidor $\mathrm{Y}$, Braunwald E, Jensen MD, Pories W, Fahrback K, Schoelies K. Bariatric surgery. A systematic review and meta-analysis. JAMA 2004; 292: 1724-37.

19. Ryden A, Torgerson JS. The Swedish obese subjects study: What has been accomplished to date? Surg Ob Rel Dis 2006; 2: 549-60.

20. Sjöström L, Narbro K, Sjöstróm D, Karason K, Larsson B, WeDEL H ET AL. Effects of bariatric surgery on mortality in Swedish obese subjects. N Engl J Med 2007; 357: 741-52.
21. Benotti PN, Forse RA. The role of gastric surgery in the multidisciplinary management of severe obesity. Am J Surg 1995; 169: 361-7.

22. Martin LF, White S, Lindstrom W. Cost-benefit analysis of the treatment of severy obesity. World J Surg 1998; 22: 1008-17.

23. BuchwaLd H. Bariatric surgery for morbid obesity: health implications for patients, health professionals, and third-party payer. J Am Coll Surg 2005; 200: 593604.

24. Flum DR, Diwinger ED. Impact of gastric bypass operation on survival: A population-based analysis. J Am Coll Surg 2004; 199: 543-51.

25. Sowemino OA, Yood SM, Courtney J, Moore J, Huang M, RESS R ET AL. Natural history of morbid obesity without surgical intervention. Surg Obes Rel Dis 2007; 3: 73-7.

26. Dixon J. Survival advantage with bariatric surgery. Report from the $10^{\text {th }}$ International Congress on Obesity. Surg Obes Rel Dis 2006; 2: 585-6.

27. Adams TD, Gress RE, Smith SC, Halmerson RCH, Simper SC, ROSAMOND WD ET AL. Long-term mortality after gastric bypass surgery. N Engl J Med 2007; 357: 75361.

28. Colquitt J, Clegg A, Sidhu M, Royle P. Surgery for morbid obesity. Cochrane Library Number CD003641, 2003.

29. North American Association for the StUdy of obesity and the National Heart, LUNG and blood institute. The practical guide: identification, evaluation and treatment of overweight and obesity in adults. $\mathrm{NIH}$ Publication 2000; 00: 4084. 